\title{
Application Analysis of Targeted Nursing Intervention for Infant Patients with Renal Dynamic Imaging
}

\author{
Lijiao Liao" ${ }^{1}$, Jinmei Xiong ${ }^{1}$, Miaoli Zhou ${ }^{1}$, Chunliu Luo ${ }^{2, *}$ \\ ${ }^{1}$ Nuclear Medicine Department, The First Affiliated Hospital of Jinan University, Guangzhou, China \\ ${ }^{2}$ Department of Medical Imaging, The First Affiliated Hospital of Jinan University, Guangzhou, China \\ Email address: \\ 272982941@qq.com (Lijiao Liao), 190598691@qq.com (Jinmei Xiong),jnuchll@163qq.com (Chunliu Luo), \\ 13622898846@163.com (Miaoli Zhou) \\ ${ }^{*}$ Corresponding author
}

\section{To cite this article:}

Lijiao Liao, Jinmei Xiong, Miaoli Zhou, Chunliu Luo. Application Analysis of Targeted Nursing Intervention for Infant Patients with Renal Dynamic Imaging. International Journal of Medical Imaging. Vol. 9, No. 1, 2021, pp. 94-97. doi: 10.11648/j.ijmi.20210901.20

Received: March 11, 2021; Accepted: March 18, 2021; Published: March 30, 2021

\begin{abstract}
Background: In recent years, nuclide renal dynamic imaging is often used to detect renal dysfunction in patients, and the imaging result will be affected by operative skills of health care personnel, patient mental health and patient Medical treatment compliance. Objective: To assess application analysis of targeted nursing intervention for infant patients with renal dynamic imaging. Methods: All patients who were between 0 and 3 years old and were scheduled to receiving renal dynamic imaging during 2019 -2020 were invited to enroll in our study. Base on receiving nursing intervention type, the patients were divided into two groups: control group and intervention group, they received common nursing intervention and targeted nursing intervention respectively. The valid data such as glomerular filtration rate, Medical treatment compliance assessments, image quality, and patients' basic information was collected from 102 infant patients with renal dynamic imaging. Result: In baseline characteristics of patient, there were no significant differences in demographics between the two groups. Intervention group had 44 cases of success in renal dynamic imaging that was significantly higher than control group, there was significant difference ( $p$ $<0.005)$. Besides, the cases of poor quality were more prevalent in control group compare with intervention group $(\mathrm{n}=14 \mathrm{vs} \mathrm{n}=$ 6). Additionally, high level assessment of Medical treatment compliance had 44 (86.3\%) cases and 30 (58.5\%) cases in intervention group and control group. Comparison result of adverse events shows that intervention group participants provide less adverse events report compare with control group [2 (3.9\%) vs $11(21.6 \%)$ ]. The adverse events of control group were present in $2(3.9 \%), 4(7.8 \%)$, and $5(9.8 \%)$ of imaging agent leaking, position skewing, insufficient imaging agent. Conclusion: targeted nursing intervention can improve quality of renal dynamic imaging, medical treatment compliance of patient, and adverse events of renal dynamic imaging for infant patients with renal dynamic imaging.
\end{abstract}

Keywords: Infant Patients, Renal Dynamic Imaging, Nursing Intervention

\section{Introduction}

In recent years, nuclide renal dynamic imaging is often used to detect renal dysfunction in patients, because function of renal dynamic imaging includes analyzing kidney size, shape, blood flow status and function [1, 2]. In Chinese hospital, doctor often use ${ }^{99 \mathrm{~m}}$ Tc-DTPA as renal dynamic imaging agent, it uses the imaging agent to inject projectile and quickly collect dynamic images to actuarial calculate the renal function status [3]. However, the imaging result will be affected by operative skills of health care personnel, patient mental health and patient Medical treatment compliance [4]. According to the above background and present situation, it is very necessary to explore the use of nursing intervention to improve imaging result of renal dynamic imaging.

Based on different research, nursing intervention improve outcome of nuclide renal dynamic imaging in patients. Especially for infant patients, they had more difficulty completing nuclide renal dynamic imaging compare with adult patient. Some studies attempt to improve outcome of 
nuclide renal dynamic imaging by nursing intervention [5-7]. Regardless of nursing intervention type, nursing intervention is considered important for improving nuclide renal dynamic imaging. For example, Qin's report shows nursing intervention improve patient satisfaction during checking of nuclide renal dynamic imaging [8]. Furthermore, the study that relevancy of nursing intervention on infant patient is worthy. The aim of this study was to evaluate application of targeted nursing for infant patients with renal dynamic imaging.

\section{Methods}

\subsection{Participants Enrollment and Survey Methods}

All patients who were between 0 and 3 years old and were scheduled to receiving renal dynamic imaging during 2019 -2020 were invited to enroll in our study. Base on receiving nursing intervention type, the patients were divided into two groups: control group and intervention group. The control group participants receive common nursing intervention in renal dynamic imaging, and the intervention group participants receive additional targeted nursing intervention based on common nursing intervention. We collected valid data from 102 infant patients with renal dynamic imaging. Of those, Medical treatment compliance assessments of participants were collected from observations of the investigator, and glomerular filtration rate, image quality, and patients' basic information were observed by medical equipment and questionnaires.

In targeted nursing intervention of this study, we used the targeted nursing intervention to improve mental health of participants, image success rate and image quality. First, participants were instructed to drink $300 \sim 500 \mathrm{ml}$ of water before examination. Then, we examined the status of the elbow veins of the participants. If the veins of the participants were too thin, we would apply a hot towel to their veins for 15 minutes and then perform venipuncture. Second, we provide participants with the knowledge of renal dynamic imaging by language, pictures, text, and video. Third, we adjust the volume and activity of the imaging agent to suitable index.

The inclusion criteria of this study contrast: (1) Age of patient was between 0 and 3 years old; (2) Patients were scheduled to receiving renal dynamic imaging; (3) Their families agreed to participate in the study. The withdraw criteria of this study contrast: (1) The patient had severe complications; (2) patients changed hospitals during the study period. (3) The patient did not complete renal dynamic imaging.

\subsection{Statistical Analysis}

We chi-square test and $t$ test to analyze the valid data from SPSS22.0. Additionally, the statistical descriptions of this study were precenting, mean and standard deviation in this paper.

\section{Result}

Of 132 individuals who completed the study screening, 102 participants met the eligibility criteria for this study. Baseline characteristics of patient are presented in Table 1. There were no significant differences in demographics between the two groups.

Table 1. Patient characteristics.

\begin{tabular}{llllll}
\hline & Age (year) & $\begin{array}{l}\text { Gender } \\
(\text { female)(n,\%) }\end{array}$ & $\begin{array}{l}\text { Course of the disease } \\
(\text { year) }\end{array}$ & $\begin{array}{l}\text { Renal related } \\
\text { diseases (case) }\end{array}$ & Other diseases (case) \\
\hline Control group $(\mathrm{n}=51)$ & $1.16 \pm 1.73$ & $17(33.3 \%)$ & $1.21 \pm 0.54$ & $44(86.3 \%)$ & $7(13.7 \%)$ \\
Intervention group $(\mathrm{n}=51)$ & $1.57 \pm 1.44$ & $18(35.3 \%)$ & $1.33 \pm 0.62$ & $30(58.9 \%)$ & $21(41.2 \%)$ \\
P value & $>0.05$ & $>0.05$ & $>0.05$ & - & - \\
\hline
\end{tabular}

The Table 2 blew shows quality of renal dynamic imaging from researcher records. Intervention group had 44 cases of success in renal dynamic imaging that was significantly higher than control group, there was significant difference $(\mathrm{p}<$
0.005). Besides, the cases of poor quality were more prevalent in control group compare with intervention group $(n=14$ vs $n$ $=6)$, and they also were significant difference $(\mathrm{p}<0.005)$.

Table 2. Quality of renal dynamic imaging.

\begin{tabular}{llll}
\hline & $\begin{array}{l}\text { Success of renal dynamic imaging } \\
\text { (case) }\end{array}$ & $\begin{array}{l}\text { Poor quality of renal dynamic } \\
\text { imaging (case) }\end{array}$ & $\begin{array}{l}\text { Failure of renal dynamic imaging } \\
\text { (case) }\end{array}$ \\
\hline Control group $(\mathrm{n}=51)$ & 35 & 14 & 2 \\
Intervention group $(\mathrm{n}=51)$ & 44 & 6 & 1 \\
$\mathrm{X}^{2}$ & 14.12 & 7.38 & 0.78 \\
$\mathrm{P}$ value & $<0.005$ & $<0.005$ & $>0.05$ \\
\hline
\end{tabular}

Table 3 displays participants' Medical treatment compliance between control group and intervention group. High level assessment of Medical treatment compliance had $44(86.3 \%)$ cases and $30(58.5 \%)$ cases in intervention group and control group, there was significant difference $(\mathrm{p}<0.005)$. Control group had more assessment cases of Middle level and low level in Medical treatment compliance compare with intervention group. Additionally, between two groups, individuals with targeted nursing intervention were significantly higher Medical treatment compliance rate than control group. 
Table 3. Comparison of Medical treatment compliance between two groups.

\begin{tabular}{lllll}
\hline & $\begin{array}{l}\text { High level of Medical } \\
\text { treatment compliance } \\
\text { (case,\%) }\end{array}$ & $\begin{array}{l}\text { Middle level of Medical } \\
\text { treatment compliance } \\
\text { (case,\%) }\end{array}$ & $\begin{array}{l}\text { Low level of Medical } \\
\text { treatment compliance } \\
\text { (case,\%) }\end{array}$ & $\begin{array}{l}\text { Medical treatment } \\
\text { compliance rate (\%) }\end{array}$ \\
\hline Control group $(\mathrm{n}=51)$ & $30(58.8 \%)$ & $13(25.5 \%)$ & $8(15.7 \%)$ & $84.31 \%$ \\
Intervention group $(\mathrm{n}=51)$ & $44(86.3 \%)$ & $4(7.8 \%)$ & $3(5.9 \%)$ & $94.1 \%$ \\
$\mathrm{X}^{2}$ & 21.95 & 5.14 & 4.45 & - \\
$\mathrm{P}$ value & $<0.005$ & $<0.05$ & $<0.05$ & - \\
\hline
\end{tabular}

In Table 4, it shows that intervention group participants provide less adverse events report compare with control group [2 (3.9\%) vs $11(21.6 \%)]$. The adverse events of control group were present in $2(3.9 \%), 4(7.8 \%)$, and $5(9.8 \%)$ of imaging agent leaking, position skewing, insufficient imaging agent. In addition, intervention group only had few adverse events cases, that include 1 case of position skewing and 1 case of insufficient imaging agent. Of those, cases of imaging agent leaking were no significant differences between the two groups, but other adverse events were significantly different between the two groups.

Table 4. Comparison of adverse events between two groups.

\begin{tabular}{llll}
\hline & $\begin{array}{l}\text { Imaging agent leaking } \\
\text { (case, } \%)\end{array}$ & Position skewing (case,\%) & $\begin{array}{l}\text { Insufficient imaging agent } \\
\text { (case, } \%)\end{array}$ \\
\hline Control group $(\mathrm{n}=51)$ & $2(3.9 \%)$ & $4(7.8 \%)$ & $5(9.8 \%)$ \\
(case, $\%)$
\end{tabular}

\section{Discussion}

We compared the quality of renal dynamic imaging, Medical treatment compliance of patient, and adverse events of renal dynamic imaging for participants of two groups. Among both control group participants and intervention group participants, the targeted nursing intervention was significantly associated with quality of renal dynamic imaging and Medical treatment compliance. Besides, the targeted nursing intervention only was associated with part of adverse events of renal dynamic imaging. Although common nursing intervention had similar association between targeted nursing intervention and renal dynamic imaging, targeted nursing intervention had better effect for renal dynamic imaging and the patients with renal dynamic imaging [9-11]. Especially for infant patients, it is more difficult to obtain high quality images in infant patients than in adult patients as they often do not cooperate with the operation of medical staffs of renal dynamic imaging [12-15]. Therefore, infant patients need the target nursing intervention which had better effect for patients in renal dynamic imaging.

Furthermore, intervention group participants had better performance in quality of renal dynamic imaging, that possible reasons are targeted nursing intervention calmed the infant patient's mood so that they are more cooperative with the operators, the results of the subsequent Medical treatment compliance evaluation also confirmed this. As for their Medical treatment compliance were improved, their adverse events were reduced in renal dynamic imaging. The possible reasons are that targeted nursing intervention are more tailored to the individual needs so that they are more effective than common nursing intervention.

\section{Conclusion}

Overall, targeted nursing intervention can improve quality of renal dynamic imaging, Medical treatment compliance of patient, and adverse events of renal dynamic imaging for infant patients with renal dynamic imaging. Although common nursing intervention also can improve those of infant patients with renal dynamic imaging, we observed better performance in the intervention group participants who received targeted nursing intervention.

\section{References}

[1] Zhu YQ. Application of Nuclide Dynamic Renal Imaging in Evaluation of Renal Function after Urinary tract Obstruction [J]. International Journal of Infectious Diseases. 2019; 8 (2): $171-172$.

[2] Gao QR. Effect of pellet injection quality on dynamic nephrography [J]. Nursing Practice \& Research. 2015; 12 (7): 124-125.

[3] GALUSKA L, BARNA S, VARGA J, et al. The role of ${ }^{99 \mathrm{~m}}$ Tc-DTPA retrobulbar SPECT in staging and follow-up of Graves' orbitopathy. Nucl Med Rev Cent East Eur, 2018, 21 (1): 54-58.

[4] Qin XY, Han XM. The application of nursing intervention in radionuclide renal dynamic imaging. Clinical Research. 2019; 27 (4): 165-166.

[5] Yuan X, Zhang J, Tang K et al. Determination of Glomerular Filtration Rate with CT Measurement of Renal Clearance of Iodinated Contrast Material versus ${ }^{99 \mathrm{~m}}$ Tc-DTPA Dynamic Imaging "Gates" Method: A Validation Study in Asymmetrical Renal Disease. Radiology. 2017; 282: 552-560. 
[6] Becker J, Babb J, Serrano M. Glomerular filtration rate in evaluation of the effect of iodinated contrast media on renal function. Am J Roentgenol. 2016; 200: 822-826.

[7] Pei XH, He J, Liu Q, Zhu B, Bao LH, Yan CJ et al. Evaluation of serum creatinine- and cystatin C-based equations for the estimation of glomerular filtration rate in a Chinese population. Scand J Urol Nephrol. 2015; 46: 223-223.

[8] Qin Xiangyin, Han Xingmin. The application of nursing intervention in patients with renal dynamic radionuclide imaging. Clinical Research. 2019; 27 (04): 165-166.

[9] Hahn OM, Yang C, Medved M, Karczmar G, Kistner E, Karrison $\mathrm{T}$, et al. Dynamic contrast-enhanced magnetic resonance imaging pharmacodynamic biomarker study of sorafenib in metastatic renal carcinoma. J Clin Oncol. 2018; 26 (28): 4572-8.

[10] Volpe A, Kachura JR, Geddie WR, Evans AJ, Gharajeh A, Saravanan A, et al. Techniques, safety and accuracy of sampling of renal tumors by fine needle aspiration and core biopsy. J Urol. 2017; 178 (2): 379-86.
[11] Tian JH, Dong H, Guo YT, Zhang Fan. Application of targeted nursing care in patients with renal dynamic radionuclide imaging. Medical Equipment. 2020; 33 (21): 140-142.

[12] Lin LY. Application effect of quality nursing intervention in patients with renal dynamic imaging. Journal of Clinical Rational Drug Use. 2019; 12 (03): 159-160.

[13] $\mathrm{Wu}$ TL. Analysis of nursing strategy in diagnosis of renal dynamic radionuclide imaging. Chin Med Guide. 2018; 16 (34): 267-268.

[14] Tan LL, Chen L, Chen X, Li XH, Wu JG, Fang XD, Li Q. Clinical significance of nursing intervention in renal dynamic imaging. Journal of Practical Medical Technology. 2017; 24 (07): 713-715.

[15] Sun MR, Ngo L, Genega EM, Atkins MB, Finn ME, Rofsky NM, et al. Renal cell carcinoma: dynamic contrast-enhanced MR imaging for differentiation of tumor subtypes-correlation with pathologic findings. Radiology. 2019; 250 (3): 793-802. 